\title{
热电材料中的晶格热导率
}

\author{
沈家骏，方腾，傅铁铮，忻佳展，赵新兵，朱铁军 \\ (浙江大学 材料科学与工程学院, 硅材料国家重点实验室, 杭州 310027)
}

摘 要: 随着可再生能源及能源转换技术的快速发展, 热电材料在发电及制冷领域的应用前景受到越来越广泛的关 注。发展具有高热电优值材料的重要性日益突出, 如何获得低晶格热导率是热电材料的研究重点之一。本文阐述了 热容、声速及弛豫时间对晶格热导率的影响, 介绍了本征低热导率热电材料所具有的典型特征, 如强非谐性、弱化 学键、本征共振散射及复杂晶胞结构等, 并分析了通过多尺度声子散射降低已有热电材料晶格热导率的方法, 其中 包括点缺陷散射、位错散射、晶界散射、共振散射、电声散射等多种散射机制。此外，总结了几种预测材料最小晶 格热导率的理论模型, 对快速笁选具有低晶格热导率的热电材料具有一定的理论指导意义。最后, 展望了如何获得 低热导率热电材料的有效途径。

关 键 词: 热电材料; 晶格热导率; 热容; 弛豫时间; 综述

中图分类号: TB34 文献标识码: A

\section{Lattice Thermal Conductivity in Thermoelectric Materials}

\author{
SHEN Jia-Jun, FANG Teng, FU Tie-Zheng, XIN Jia-Zhan, ZHAO Xin-Bing, ZHU Tie-Jun
}

(State Key Laboratory of Silicon Materials, School of Materials Science and Engineering, Zhejiang University, Hangzhou 310027, China)

\begin{abstract}
With rapid development of sustainable energies and energy conversion technologies, application prospect of thermoelectric (TE) materials in power generation and cooling has received increasing attention. The requirement of improving TE materials with high figure of merit becomes much more important. How to obtain the low lattice thermal conductivity is one of the main concerns in TE materials. In this review, the influences of specific heat, phonon group velocity and relaxation time on the lattice thermal conductivity are discussed, respectively. Several typical features of TE materials with intrinsic low lattice thermal conductivity are introduced, such as strong anharmonicity, weak chemical bonds and complex primitive cells. Introducing multiscale phonon scatterings to reduce the lattice thermal conductivity of known TE materials is also presented and discussed, including but not limited to point defect scattering, dislocation scattering, boundary scattering, resonance scattering and electron-phonon scattering. In addition, some theoretical models of the minimum lattice thermal conductivity are analyzed, which has certain theoretical significance for rapid screening of TE materials with low lattice thermal conductivity. Finally, the efficient ways to obtain the low lattice thermal conductivity for TE property optimization are proposed.
\end{abstract}

Key words: thermoelectric materials; lattice thermal conductivity; specific heat; relaxation time; review

热电材料是一种可以实现热能与电能直接相互 转化的能源材料，可用于废热发电、制冷等领域 ${ }^{[1]}$ 。

收稿日期: 2018-07-16; 收到修改稿日期：2018-09-03

基金项目: 国家自然科学基金(51725102,51761135127, 11574267)

National Natural Science Foundation of China (51725102, 51761135127, 11574267)

作者简介: 沈家骏(1992-), 男, 博士研究生. E-mail: 11626058@zju.edu.cn

通讯作者: 朱铁军, 教授. E-mail: zhutj@zju.edu.cn 
相比于传统热机或者压缩机, 热电材料制成的器件 具有结构简单、空间占用率小、无机械运动等优点 ${ }^{[2]}$ 。 热电转化效率低是目前制约热电器件广泛应用的主 要因素。热电材料的转化效率主要取决于材料的热 电性能, 衡量热电性能高低的指标称为热电优值, $Z T=\frac{S^{2} \sigma}{\kappa} T$, 其中 $T$ 为绝对温度, $S$ 为塞贝克系数, $\sigma$ 为电导率, $\kappa$ 为热导率。因此高热电优值成为提高热 电转化效率的关键。但是, 影响热电优值的三个参 数通过载流子浓度相互耦合 ${ }^{[3]}$, 获得高热电优值面 临诸多挑战。

热电优值优化的常用方法是在优化载流子浓度 成分附近尽可能降低材料热导率。热导率 $\kappa$ 又分为 电子热导率 $\kappa_{\mathrm{e}}$ 和晶格热导率 $\kappa_{\mathrm{L}}$ 。电子热导率服从魏 德曼-弗兰茨定律 $\kappa_{\mathrm{e}}=L \sigma \mathrm{T}$, 其中 $L$ 为洛伦茨常数。 可以看出, 电子热导率与电导率呈正比关系。晶格 热导率是一个可以相对独立调控的参数, 因而, 降 低晶格热导率成为优化热电优值的有效手段 ${ }^{[4-9]}$ 。基 于理想气体模型假设, 晶体中的晶格热导率可表示 为 $\kappa_{\mathrm{L}}=\frac{1}{3} C_{\mathrm{V}} v_{\mathrm{g}} l=\frac{1}{3} C_{\mathrm{V}} v_{\mathrm{g}}^{2} \tau$, 其中 $C_{\mathrm{V}}$ 为定容热容, $v_{\mathrm{g}}$ 为 声子振动模的群速度, $l$ 为平均自由程, $\tau$ 为声子弛豫 时间。降低任意一个参数均可以降低晶格热导率。 本文首先从热容、声速及弛豫时间三个角度阐述降 低热导率的策略, 然后对最小晶格热导率的计算模 型进行简单介绍, 最后总结获得低热导率热电材料 的有效途径与方法。

\section{1 晶格热导率的影响因素}

\section{1 热容}

从晶格热导率的公式可以看出, 晶格热导率与 定容热容 $C_{\mathrm{V}}$ 成正比, 所以降低 $C_{\mathrm{V}}$ 有利于获得低晶 格热导率。杜隆一珀蒂定律指出构成固体的各个原子 在高温时的热容趋近于极限值 $3 k_{\mathrm{B}}$, 其中 $k_{\mathrm{B}}$ 为玻尔 兹曼常数, 因此通过改变热容来降低热导率通常比 较困难。然而研究人员发现中温 $\mathrm{Cu}_{2-x} \mathrm{Se}$ 热电材料 的热容随着温度升高会逐渐减小 ${ }^{[10-11]}$ 。如图 1(a)所 示, 固体的 $C_{\mathrm{V}}$ 为 $3 N k_{\mathrm{B}}, N$ 为总原子数。而在液体中, 大部分横向振动波无法传递, $C_{\mathrm{V}}$ 减小到 $2 \sim 2.5 N k_{\mathrm{B}}$ 。 随着温度升高, $\mathrm{Cu}_{2-x} \mathrm{Se}$ 中 $\mathrm{Cu}$ 离子的高度离域性使 材料在一定程度上表现出类液体的行为, 大大降低 了横向声学波对热容的贡献, 使得 $\mathrm{Cu}_{2-x} \mathrm{Se}$ 在高温 条件下的 $C_{\mathrm{V}}$ 接近 $2 N k_{\mathrm{B}}$ 。

根据德拜热容模型, 热导的贡献主要来自于声 学波。这是由于光学波的波速趋近于 0 , 对热导率的
贡献基本可以忽略 ${ }^{[17]}$ 。对于多原子体系，假设每个 原胞中的原子数为 $n$, 总原胞数为 $N$, 则体系总的自 由度为 $3 n N$ 。其中, 声学支有 $3 N$ 个, 光学支有 $3(n-1) N$ 个 ${ }^{[18]}$ 。根据能量均分原理可得, 声学支贡献 的热容为 $C / n$, 光学支为 $C(n-1) / n$ 。因此, 原胞中原 子数越多, 声学支对总热容的贡献越小, 越有利于 获得低晶格热导率。这在许多具有复杂晶胞结构的 热电材料中得到了印证(如图 1(b)所示)。例如: $\mathrm{Yb}_{14} \mathrm{MnSb}_{11}$ 与 $\mathrm{PbTe}$ 具有相似的德拜温度, 平均原 子质量, 热容及格林内森参数 ${ }^{[19-20]}$, 但是由于 $\mathrm{Yb}_{14} \mathrm{MnSb}_{11}$ 原胞中原子数为 104 , 而 $\mathrm{PbTe}$ 只有 2 , 使 得 $\mathrm{Yb}_{14} \mathrm{MnSb}_{11}$ 的室温晶格热导率仅有 $0.6 \mathrm{~W} \cdot \mathrm{m}^{-1} \cdot \mathrm{K}^{-1}$, 远低于 $\mathrm{PbTe}$ 的 $2 \mathrm{~W} \cdot \mathrm{m}^{-1} \cdot \mathrm{K}^{-1[21]}$ 。

\section{2 声速}

在热导率公式中, $v_{\mathrm{g}}$ 为声子的群速度，可以根 据声子谱中声学支的斜率得到。在实际研究中, 为 了便于测量, 通常假设群速度 $v_{\mathrm{g}}$ 与固体声速 $v_{\mathrm{s}}$ 相 等。研究表明具有弱化学键的化合物通常具有低声 速,例如,在 $\alpha-\mathrm{MgAgSb}$ 中由于 $\mathrm{Mg}$ 原子本身不存在 $\mathrm{d}$ 轨道, 无法与近邻原子形成强 $\mathrm{d}-\mathrm{d}$ 键, 导致 $\mathrm{Mg}$ 原子与 $\mathrm{Ag}$ 原子之间的共价键较弱, 晶格发生一定的扭曲 ${ }^{[22]}$ 。 这种弱键的存在使得 $\alpha-\mathrm{MgAgSb}$ 的声速仅有 $1920 \mathrm{~m} / \mathrm{s}$ 。
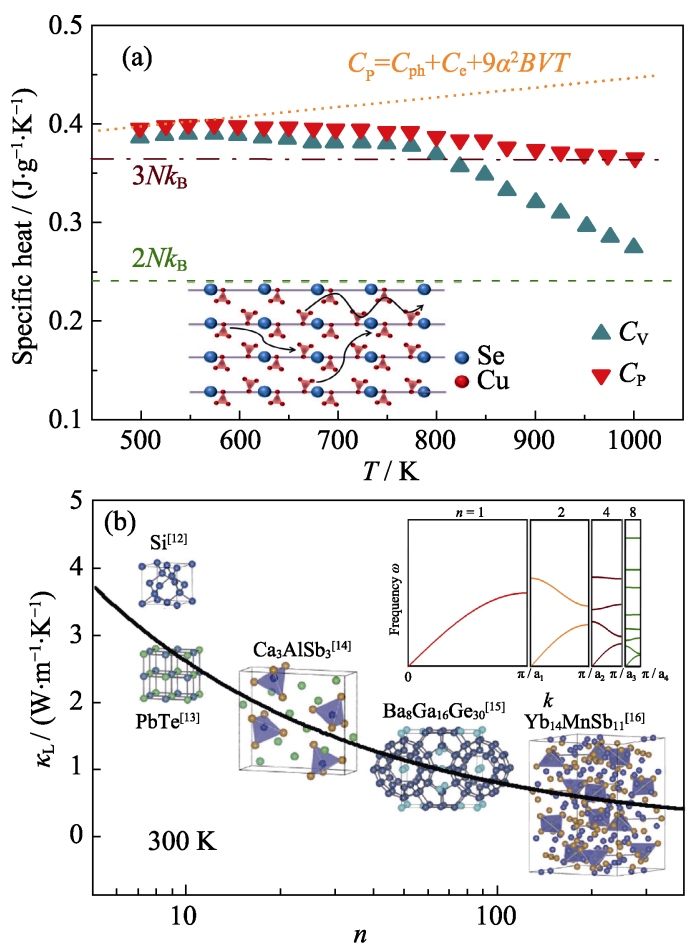

图 1 (a) $\mathrm{Cu}_{2-x} \mathrm{Se}$ 化合物的热容与温度关系图 ${ }^{[11]}$ 和(b) 室温 晶格热导率与原胞中原子数关系 ${ }^{[12-16]}$

Fig. 1 (a) Temperature dependence of the lattice thermal conductivity for $\mathrm{Cu}_{2-x} \mathrm{Se}^{[11]}$ and (b) number of atoms in the primitive unit cell versus room temperature lattice thermal conductivity $^{[12-16]}$ 
$\mathrm{Ag}_{8} \mathrm{GeTe}_{6}$ 等体系中也存在同样的现象 ${ }^{[23-24]}$, 由于 $\mathrm{Ag}$ 原子与 $\mathrm{Te}$ 原子之间较弱的键合使得 $\mathrm{Ag}_{8} \mathrm{GeTe}_{6}$ 声速低 至 $1000 \sim 1500 \mathrm{~m} / \mathrm{s}$ 。

此外, 通过引入重原子的方式也能降低声速, 比 较典型的例子有笼式化合物及填充方钴矿 ${ }^{[25-28]}$ 。如 图 2(a)所示, 在 $\mathrm{Ba}_{8} \mathrm{Ga}_{16} \mathrm{Ge}_{30}$ 笼式化合物中, $\mathrm{Ba}$ 原子 占据由 $\mathrm{Ga}$ 原子及 $\mathrm{Ge}$ 原子构成的晶格间隙中。若将 $\mathrm{Ba}$ 原子单纯看作散射中心, 则理论预测的声子弛豫 时间应为 $0.18 \mathrm{ps}$ 。然而, 中子三轴光谱分析表明加 入 $\mathrm{Ba}$ 原子仅仅使纵波弛豫时间 $\tau_{\mathrm{L}}$ 由 $2.6 \mathrm{ps}$ 降为 $1.3 \mathrm{ps}$ 。因此, 低晶格热导率不能只归因于弛豫时间 的降低, 声速的降低也起到了重要的作用。如图 2(b) 和(c)所示, 在笼式结构中, 由于声学声子模与填充 原子产生的低频光学支之间存在 “避免交叉” 现象, 使得声学支的频率进一步降低, 从而具有更低的声 速 ${ }^{[29]}$ 。

\section{3 弛豫时间}

根据马希森定则, 如果材料中存在多种散射 机制, 则声子的总弛豫时间可写成如下形式: $\tau^{-1}=\tau_{\mathrm{U}}^{-1}+\tau_{\mathrm{PD}}^{-1}+\tau_{\mathrm{B}}^{-1}+\tau_{\mathrm{Str}}^{-1}+\tau_{\mathrm{Res}}^{-1}+\tau_{\mathrm{EP}}^{-1}+\cdots$, 其中 $\tau_{\mathrm{U}}$ 、 $\tau_{\mathrm{PD}} 、 \tau_{\mathrm{B}} 、 \tau_{\mathrm{Str}} 、 \tau_{\mathrm{Res}} 、 \tau_{\mathrm{EP}}$ 分别为 $\mathrm{U}$ 过程散射、点 缺陷散射、晶界散射、位错散射、非磁性声子共振 散射以及电声散射引起的弛豫时间。对于固体材料, 其内部的热传输声子分布在一个较宽的频率范围内, 因此要获得较低的晶格热导率, 则需要对不同频率 的声子进行散射 ${ }^{[30-31]}$ 。

$\mathrm{U}$ 过程散射是材料固有的本征散射。在高温极 限条件下, $\mathrm{U}$ 过程散射是主要散射机制, 晶格热导 率 $\kappa_{\mathrm{L}} \propto 1 / T$ 。U 过程散射的强弱可通过 Slack 公式
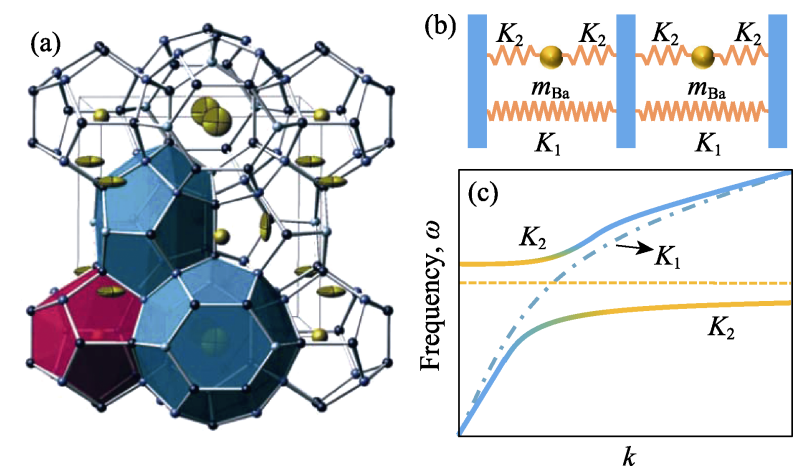

图 2 (a) $\mathrm{Ba}_{8} \mathrm{Ga}_{16} \mathrm{Ge}_{30}$ 晶体结构示意图, (b) 未填充及填充笼式 结构的弹簧模型及 (c)色散关系 ${ }^{[29]}$

Fig. 2 (a) Schematic diagram of crystal structure for $\mathrm{Ba}_{8} \mathrm{Ga}_{16} \mathrm{Ge}_{30}$, (b) a simple spring model and (c) the corresponding dispersion relation of filled and unfilled clathrate ${ }^{[29]}$ describing interaction between the host cages with a spring constant $K_{1}$ and the guest atoms attached to the cages with a spring constant $K_{2}$
$\tau_{\mathrm{U}}^{-1} \approx \frac{\hbar \gamma^{2}}{M v^{2} \theta_{\mathrm{D}}} \omega^{2} T \exp \left(-\theta_{\mathrm{D}} / 3 T\right)^{[32]}$ 判断, 式中 $\gamma$ 为格林 内森常数, $M$ 为平均原子质量, $v$ 为声速, $\theta_{\mathrm{D}}$ 为德拜 温度, $\omega$ 为声子频率。在这些参数中, 声速与德拜温 度均与化学键强弱有关, 而格林内森常数则表征化 学键的非谐性强弱 ${ }^{[33]}$ 。格林内森常数越大, 则非谐 作用越强, 晶格热导率越低(如图 3 所示)。研究表明, 具有高格林内森常数的材料一般具有以下两个特 征: 晶体非对称性和存在光声耦合作用。 $\mathrm{Cu}_{3} \mathrm{SbSe}_{3}$ 中 $\mathrm{Sb}$ 原子具有孤对电子, 使 $\mathrm{Cu}_{3} \mathrm{SbSe}_{3}$ 晶格结构的 对称性遭到破坏, 具有较高的格林内森常数 ${ }^{[34-35]}$ 。 传统的中温区热电材料 $\mathrm{PbTe}$ 具有较高的格林内森 常数 $(\sim 1.4)^{[36-37]}$ 。这是由于在 $\mathrm{PbTe}$ 材料中, 纵声学 波和横光学波之间存在强烈的耦合作用, 从而大大 降低了 $\mathrm{PbTe}$ 材料的晶格热导率 ${ }^{[38]}$ 。

点缺陷散射是一种非常有效的降低材料热导率 的方法, 包括质量波动散射与应力场波动散射 ${ }^{[54]}$, 二 者分别与原子间质量差和半径差有关, 原子间质量差 及半径差越大，点缺陷散射越强。合金化是目前应用 最广的增强点缺陷散射的手段, 在 $\mathrm{Bi}_{2} \mathrm{Te}_{3}{ }^{[55-56]}$ 、 $\mathrm{Pb}(\mathrm{Te}, \mathrm{Se})^{[57]} 、 \mathrm{CuInTe}_{2}{ }^{[58]} 、 \mathrm{Mg}_{2}(\mathrm{Si}, \mathrm{Sn})^{[59-60]} 、 \mathrm{SiGe}$ 合 金 ${ }^{[61]}$ 以及 Half-Heusler $(\mathrm{HH})$ 合金 ${ }^{[62-69]}$ 中都有应用。 以 $\mathrm{FeNbSb}$ 基 $\mathrm{HH}$ 合金为例, 研究表明 $\mathrm{Nb}$ 位 $\mathrm{Ta}$ 合金 化可以有效降低 FeNbSb 的晶格热导率 ${ }^{[70]}$ 。如图 4(a) 及(b)所示, 虽然 $\mathrm{Nb}$ 和 $\mathrm{Ta}$ 之间较小的原子半径差使 应力场波动散射较弱, 但两者较大的原子质量差可 以引入强烈的质量波动散射, 使其最小晶格热导率 降至 $1.3 \mathrm{~W} \cdot \mathrm{m}^{-1} \cdot \mathrm{K}^{-1}$ 。除了合金化之外, 空位与间隙 原子也属于一种较为特殊的点缺陷散射机制。1 9 电 子 $\mathrm{HH}$ 合金 $\mathrm{Nb}_{0.8} \mathrm{CoSb}$ 中存在近 $20 \%$ 的本征 $\mathrm{Nb}$ 空位, 使 $\mathrm{Nb}_{0.8} \mathrm{CoSb}$ 合金具有相对较低的晶格热导率 ${ }^{[71]}$ 。 $\mathrm{Cu}_{2} \mathrm{SnSe}_{4}$ 等热电材料中也存在同样的现象 ${ }^{[72]}$ 。

晶界散射及位错散射也是非常重要的降低晶格

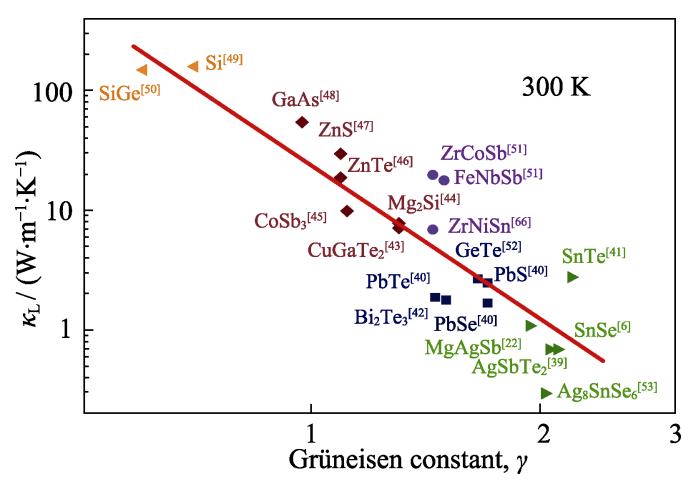

图 3 格林内森常数与室温晶格热导率的关系图 ${ }^{[6,22,39-53]}$

Fig. 3 Grüneisen parameter versus room temperature lattice thermal conductivity ${ }^{[6,22,39-53]}$ 

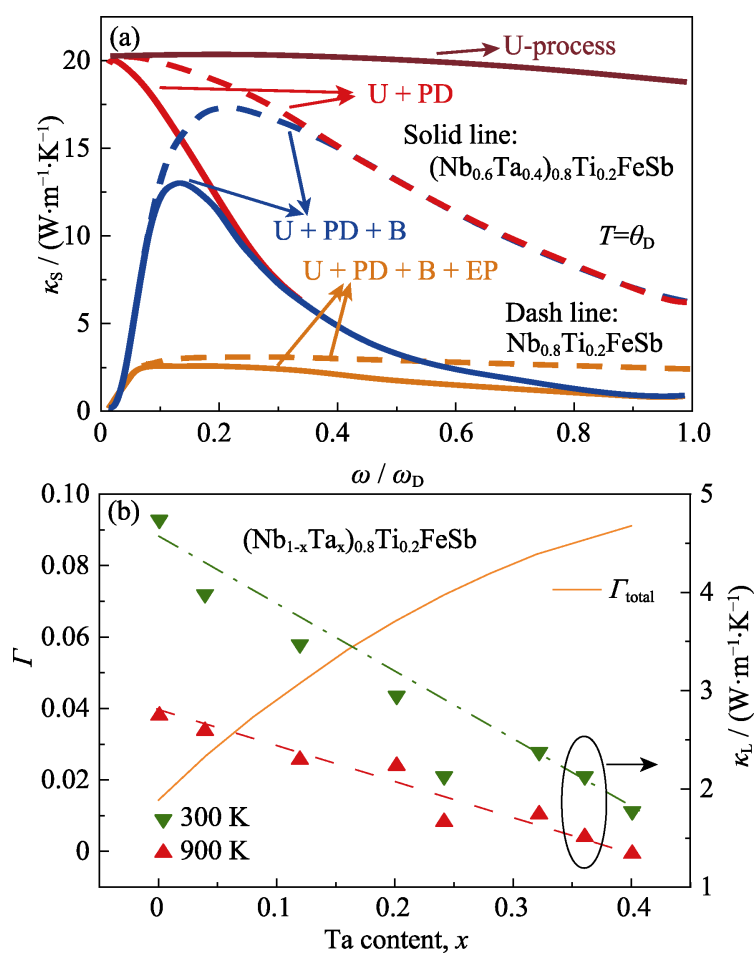

图 4 (a) $\left(\mathrm{Nb}_{0.6} \mathrm{Ta}_{0.4}\right)_{0.8} \mathrm{Ti}_{0.2} \mathrm{FeSb}$ 和 $\mathrm{Nb}_{0.8} \mathrm{Ti}_{0.2} \mathrm{FeSb}$ 的晶格热导 率与声子频率的依赖关系和(b)Ta 掺杂量与无序散射因子及 晶格热导率的关系图 ${ }^{[70]}$

Fig. 4 (a) Phonon frequency dependence of spectral lattice thermal conductivity for $\left(\mathrm{Nb}_{0.6} \mathrm{Ta}_{0.4}\right)_{0.8} \mathrm{Ti}_{0.2} \mathrm{FeSb}$ and $\mathrm{Nb}_{0.8} \mathrm{Ti}_{0.2} \mathrm{FeSb}$, and (b) relationship between Ta content and lattice thermal conductivity/disorder parameter for $\left(\mathrm{Nb}_{0.6} \mathrm{Ta}_{0.4}\right)_{0.8} \mathrm{Ti}_{0.2} \mathrm{FeSb}^{[70]}$

热导率的方法 ${ }^{[73]}$ 。常用的增强晶界散射的手段有球 磨 ${ }^{[74-75]}$ 和甩带 ${ }^{[76-79]}$ 两种方法, 广泛应用于 $\mathrm{Bi}_{2} \mathrm{Te}_{3}{ }^{[79]}$ 、 $\mathrm{SiGe}^{[80]}$ 以及 Half-Heusler 合金 ${ }^{[81]}$ 等热电材料中。对 于位错散射，有报道称在 $\mathrm{Bi}_{0.5} \mathrm{Sb}_{0.15} \mathrm{Te}_{3}$ 中通过过量 $\mathrm{Te}$ 液相烧结的手段可以增加晶界位错阵列 ${ }^{[8]}$ 。另外, 通过向材料中添加第二相的方式也能增加位错密度 ${ }^{[82]}$, 这一现象在 $\mathrm{PbTe}-\mathrm{PbS}$ 体系中得到了印证 ${ }^{[83]}$ 。

此外，位错还可以通过自身空位的聚集产生。 如图 5 所示, 在 $\mathrm{Mg}_{2} \mathrm{Si}_{1-x} \mathrm{Sb}_{x}$ 材料 ${ }^{[84]}$ 中, $\mathrm{Sb}$ 的高剂量 合金化产生大量 $\mathrm{Mg}$ 空位，使得空位浓度远高于平 衡空位密度, 多余的 $\mathrm{Mg}$ 空位自发地发生聚集从而 形成位错。在 $\mathrm{Mg}_{2} \mathrm{Si}_{0.5} \mathrm{Sb}_{0.5}$ 中, 位错密度高达 $2.8 \times 10^{16} \mathrm{~m}^{-2}$ 。图 5(b)为 $\mathrm{Sn}$ 及 $\mathrm{Sb}$ 元素合金化对 $\mathrm{Mg}_{2} \mathrm{Si}$ 晶格热导率的不同影响。由于 $S n$ 元素的加入不会增 加 $\mathrm{Mg}$ 空位的浓度从而产生位错, 晶格热导率的降 低主要来自点缺陷散射的作用。而 $\mathrm{Sb}$ 合金化不仅能 增强点缺陷散射, 而且能增强位错散射, 因此具有 更低的晶格热导率。此外, 在 $\mathrm{Na}_{y} \mathrm{Eu}_{0.03} \mathrm{~Pb}_{0.97-y} \mathrm{Te}$ 体 系 ${ }^{[7]}$ 中也观察到类似的现象。研究表明随着 $\mathrm{Na}$ 掺杂 量的增加, 体系中的主要微观缺陷由点缺陷逐步过 渡到位错及纳米颗粒。位错散射使 $\mathrm{PbTe}$ 的晶格热导 (a)
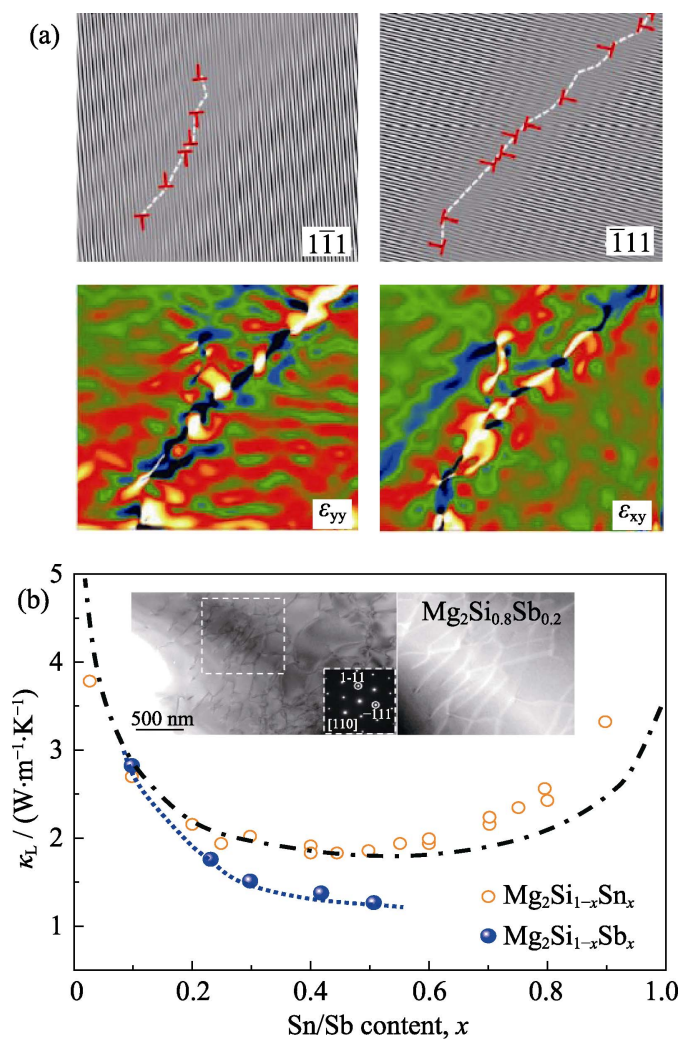

图 5 (a) $\mathrm{Mg}_{2} \mathrm{Si}_{0.5} \mathrm{Sb}_{0.5}$ 中位错的 IFFT 图及相应的应力扫描 图, (b) $\mathrm{Mg}_{2} \mathrm{Si}_{1-x} \mathrm{Sb}_{x}$ 及 $\mathrm{Mg}_{2} \mathrm{Si}_{1-z} \mathrm{Sn}_{z}$ 的室温晶格热导率对比图 ${ }^{[84]}$ Fig. 5 (a) Inverse FFT images and strain mapping of dislocations in the $\mathrm{Mg}_{2} \mathrm{Si}_{0.5} \mathrm{Sb}_{0.5}$, and (b) lattice thermal conductivity comparison between $\mathrm{Mg}_{2} \mathrm{Si}_{1-x} \mathrm{Sb}_{x}$ and $\mathrm{Mg}_{2} \mathrm{Si}_{1-z} \mathrm{Sn}_{z}$ at room temperature $^{[84]}$

率下降到 $0.4 \mathrm{~W} \cdot \mathrm{m}^{-1} \cdot \mathrm{K}^{-1}$ 以下。

除此之外, 共振散射一般出现在具有特殊晶体结

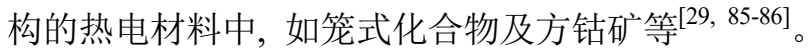
通过加入填充原子, 可以引入特定频率的共振谱, 从而降低材料热导率。在 $\mathrm{S}_{0.5} \mathrm{Co}_{4} \mathrm{Sb}_{10.5} \mathrm{Te}_{1.5}$ 中 $\mathrm{S}$ 作为 填充原子, 可以在声子谱中引入一段频率较低的光 学支, 与声学支发生光声耦合现象, 增强共振散射, 最终使得 $\mathrm{CoSb}_{3}$ 材料获得极低的晶格热导率 ${ }^{[87]}$ 。另 外, 最近的研究表明一些具有拓扑绝缘性的热电材 料中也存在共振散射。如图 6 所示, 在 BiSe 材料中 额外的 $\mathrm{Bi}_{2}$ 原子层同样可以引起局域共振效应，强 烈的光声耦合显著降低了 $\mathrm{BiSe}$ 的晶格热导率，使其 室温晶格热导率仅为 $0.6 \mathrm{~W} \cdot \mathrm{m}^{-1} \cdot \mathrm{K}^{-1[88]}$ 。

相比于其他类型的散射机制, 电声散射的研究 相对较少。然而, 对于具有较大载流子有效质量的 体系, 通常需要较高的载流子浓度使其电性能达到 最佳 ${ }^{[89-92]}$ 。因此, 在这些体系中需要考虑电声散射 对晶格热导率的影响。如图 7 所示, 在多晶硅中掺 入 $\mathrm{P}$ 使其晶格热导率显著降低, 0.1 at $\%$ 的 $\mathrm{P}$ 掺杂量就 可以使多晶硅室温时的晶格热导率降低 $60 \%$ 。但由 

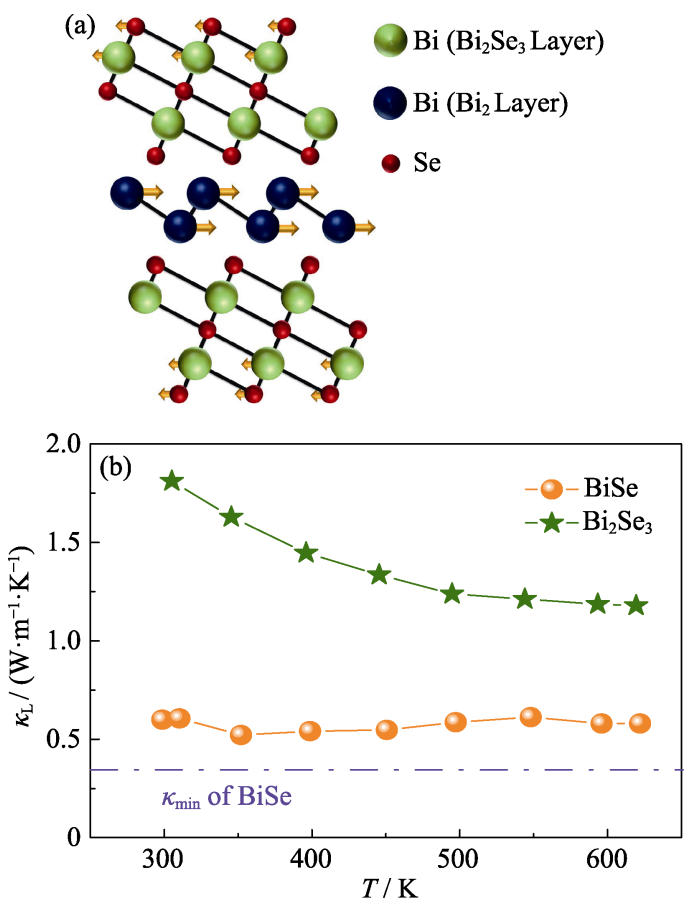

图 $6 \mathrm{BiSe}$ 晶体结构示意图(a)和 $\mathrm{Bi}_{2} \mathrm{Se}_{3}$ 及 $\mathrm{BiSe}$ 的晶格热导 率对比图(b) ${ }^{[88]}$

Fig. 6 (a) Schematic diagram of crystal structure for BiSe and (b) lattice thermal conductivity comparison between $\mathrm{Bi}_{2} \mathrm{Se}_{3}$ and $\mathrm{BiSe}^{[88]}$
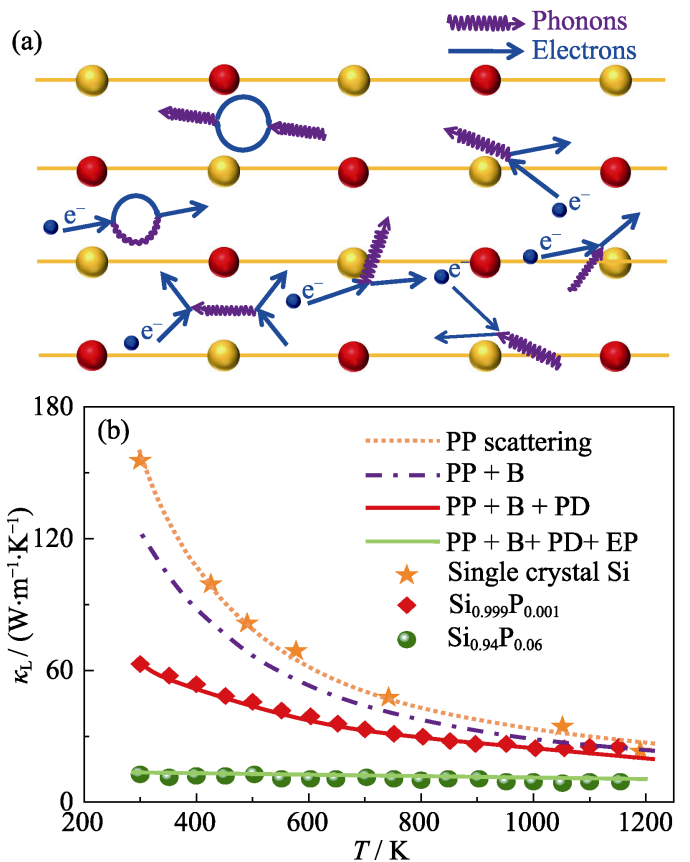

图 7 电声散射示意图(a)和硅样品晶格热导率的实验值与 Callaway 模型计算值的对比图(b) ${ }^{[93]}$

Fig. 7 (a) Schematic diagram of electron-phonon scattering and (b) comparison of experimental and calculated lattice thermal conductivities by Callaway Model for the silicon sample ${ }^{[93]}$

于 $\mathrm{P}$ 与 $\mathrm{Si}$ 在元素周期表中的位置接近, 具有相似的 质量和半径, 因此点缺陷散射不足以解释其晶格热 导率的大幅度降低，必然存在其他散射机制的作
用。研究表明 P 元素的掺杂作用可以引起多晶硅中 载流子浓度的增加，使电声相互作用显著增强。当 掺入 $6 a t \%$ 的 P 时, 电声散射对晶格热导率的降低作 用占所有散射机制的 $36 \%$ ，接近晶界散射的作用 ${ }^{[93]}$ 。

\section{2 最小晶格热导率}

为了能够快速篮选具有低晶格热导率的热电材 料, 采用合理模型预测理论最小晶格热导率是非常 必要的。上文提到, 晶格热导率的表达式可近似为 $\kappa_{\mathrm{L}}=\frac{1}{3} C_{\mathrm{V}} v_{\mathrm{g}} l$ 。由于一般热电材料达到最小晶格热导 率时都已经高于德拜温度, 此时热容已经趋近于杜 隆-珀蒂值 $3 R$ 。代入晶格热导率公式可得: $\kappa_{\mathrm{L}}=R v_{\mathrm{g}} l$, 其中 $R$ 为理想气体常数。因此, 想要获得最小晶格 热导率, 声速及平均自由程需要达到最小值。这里 需要注意的是由于热容公式中只考虑了声学支对热 导率的贡献, 造成理论结果低于实际情况 ${ }^{[94]}$ 。由于 平均声速可通过测试材料的弹性模量与密度获得, 因此如果知道最小声子平均自由程就可以计算材料 的最小晶格热导率。Clarke 等 ${ }^{[95]}$ 提出最小声子平均 自由程等于原胞体积的三次方根, 此时最小晶格热 导率的表达式如下: $\kappa_{\text {min }}=0.93 n^{2 / 3} k_{\mathrm{B}} \frac{1}{3}\left(2 v_{\mathrm{S}}+v_{\mathrm{P}}\right)$ 。其 中, $n$ 为原胞中原子数。Cahill 等 ${ }^{[96]}$ 和 Slack $^{[17]}$ 采用
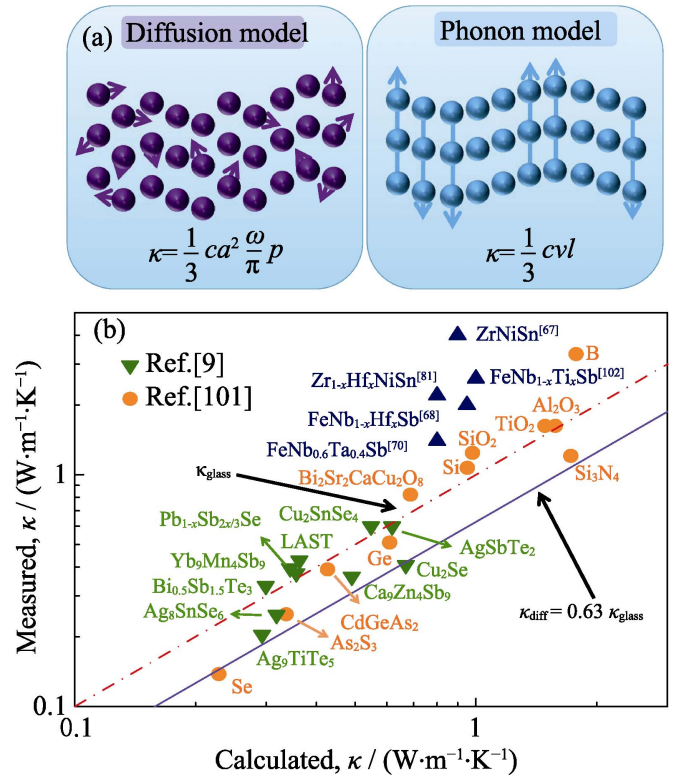

图 8 (a)扩散子模型及声子模型的差别示意图和(b)Cahill 模 型及扩散子模型预测的最小晶格热导率对比图

Fig. 8 (a) Schematic diagram of the difference between diffusion model and phonon model, and (b) comparison of calculated minimum lattice thermal conductivities by Cahill model and diffuson model 
Einstein 模型 ${ }^{[97]}$ 计算材料处于非晶态时的最小晶格 热导率。但是, 在处理热容问题时, Einstein 模型与 实验结果之间存在较大偏离。为了避免 Einstein 热 容的缺陷, Cahill 在 Einstein 假设的基础上将若干个 原子视为一个整体, 作为单个振子处理。这种处理 方式保留了晶格的周期性特征。Cahill 假设最小声 子平均自由程为声子波长的一半。

虽然最小晶格热导率的计算模型有所不同，但 不同模型预测出的结果大都比较接近。这主要由两 方面原因造成的: 首先, 大多数材料达到最小晶格 热导率时, 温度均远远高于德拜温度, 此时, 热容 已经趋近于极限值 $3 R$; 其次, 所有模型都假设对热 导率的贡献全部由平均自由程等于原子间距尺度的 声子贡献, 这一假设使得晶体中尺度大于原子间距 的缺陷, 如位错、第二相等不会对最小晶格热导率 的预测产生影响。

不管是 Cahill 模型还是 Clarke 模型, 其本质都 是基于声子模型及气体碰撞模型推导而得到的。然
而，对于某些材料来说，实验能够得到的最小晶格 热导率低于理论预测值, 是由于声子的数学描述要 求晶格具有周期性，而对于非晶、纳米晶及准晶材 料来说，晶格并不具有周期性。Allen 及 Feldman 提 出了对于这种具有非周期性结构材料的最小晶格热 导率计算模型一扩散子模型 ${ }^{[98-99]}$ 。图 8(a)显示了扩 散子模型及声子模型之间的差别，这一模型不再将 晶格振动看作传递能量的声子, 而是符合随机漫步 理论的扩散子。扩散子模型的能量传递方式由扩散 控制，该模型下晶格热导率的表达式可以表示为:

$\kappa_{\text {diff }}=0.76 n^{2 / 3} k_{\mathrm{B}} \frac{1}{3}\left(2 v_{S}+v_{\mathrm{P}}\right)$ 。通过扩散子模型计算的 最小晶格热导率比 Cahill 模型的最小晶格热导率低约 $37 \%{ }^{[100]}$ 。研究表明对于具有无序结构或者复杂原胞的 材料来说，这一模型更加适用。如图 8(b)所示, Cahill 模型的计算值大体与实验室所能获得的最小晶格 热导率值相当。而扩散控制的最小晶格热导率则能 更好地表征一种材料所能达到的极限晶格热导率。
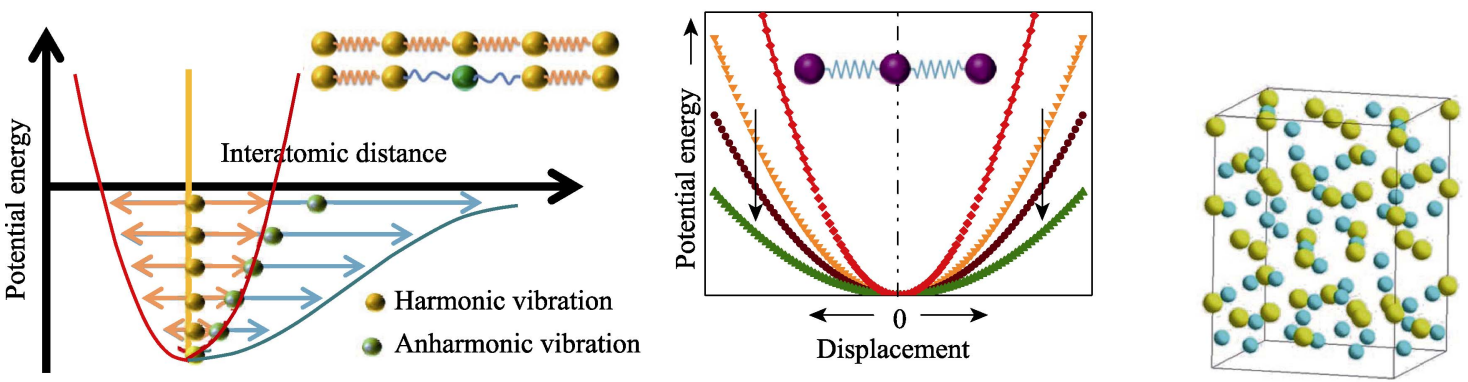

Weak chemical bonds

Strong anharmonicity
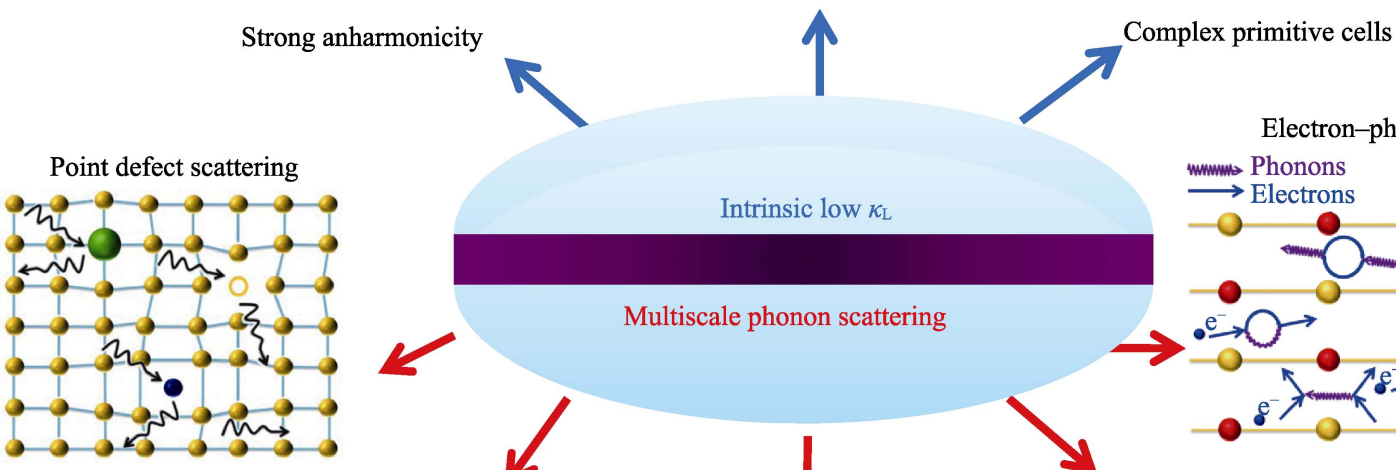

Boundary scattering
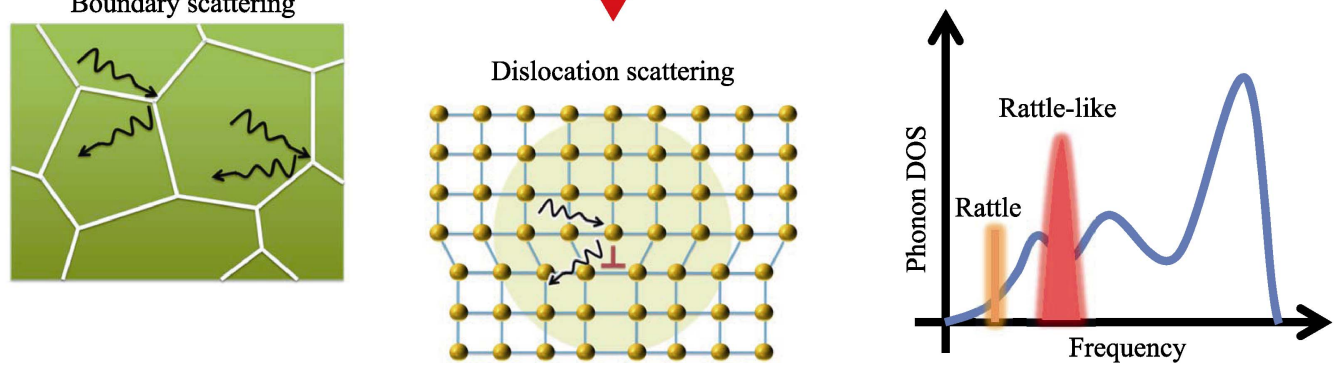

图 9 获得低晶格热导率的几种途径

Fig. 9 Several strategies to obtain low lattice thermal conductivity 


\section{3 结束语}

晶格热导率是一个可以相对独立调控的影响材 料热电性能的参数。本文分别阐述了热容、声速及 弛豫时间等三个物理量对晶格热导率的影响, 并介 绍了几种不同类型的预测材料最小晶格热导率的理 论模型, 对降低材料的晶格热导率具有重要的指导 意义。那么如何从实验上获得较低的晶格热导率呢? 主要可以从以下两方面考虑:

第一、寻找并制备具有本征低热导率的热电材 料。具有本征低热导率的热电材料一般具有以下几 个特征: 1. 强非谐性。非谐性强弱主要与化学键及 原子平衡位置的对称性有关。原子在振动过程中, 若 其对称中心发生偏移越大, 则非对称性越强。具有孤 对电子的材料往往由于电子云分布不均匀, 晶体结 构会发生一定的变形, 非对称性显著增强, 有利于获 得强非谐性; 2. 弱化学键。化学键弱的材料具有较低 的声速, 原子在其平衡位置附近具有更大的活动空 间, 电子云分布更为弥散。在声子谱中, 弱化学键往 往对应一些低频段的声子模, 更容易与声学支发生 耦合作用, 从而进一步降低声学支对热导的贡献; 3 . 复杂的晶胞结构。一方面可以降低声学支对总热容 的贡献比重, 另一方面可以降低声学支声子的群速。

第二、通过多尺度声子散射降低已有热电材料 的热导率。由于在德拜温度以上，声子频率分布在 0 到德拜频率之间, 同时抑制所有波长段的声子模能 够有效降低晶格热导率, 如点缺陷散射、位错散射、 晶界散射、共振散射和电声散射等(如图 9 所示)。

近年来有研究表明, 弱拓扑绝缘体能实现极低 的晶格热导率 ${ }^{[101-103]}$, 并且其特殊的表面传导特性有 望冲破半导体基热电材料的禁锢, 实现电性能及热性 能的真正解耦。然而, 拓扑绝缘体的晶格动力学、声 子输运等机制仍需要人们进一步研究与探索 ${ }^{[104-105]}$ 。 总的来说, 不论是研究发现新型的具有本征低晶格 热导率的热电材料, 还是对现有的热电材料热导率 进一步的降低, 通过多种手段的并用, 一定会对未 来的热电材料领域的可持续发展产生实质的积极促 进作用。

\section{参考文献:}

[1] BELL L E. Cooling, heating, generating power, and recovering waste heat with thermoelectric systems. Science, 2008, 321(5895): 1457-1461.

[2] SNYDER G J, TOBERER E S. Complex thermoelectric materials. Nature Materials, 2008, 7: 101-110.

[3] XIN J Z, TANG Y L, LIU Y T, et al. Valleytronics in thermoelectric materials. npj Quantum Materials, 2018, 3(1): 9.
[4] LI W, ZHENG L L, GE B H, et al. Promoting SnTe as an eco-friendly solution for $\mathrm{p}-\mathrm{PbTe}$ thermoelectric via band convergence and interstitial defects. Advanced Materials, 2017, 29(17): 1605887-1-8.

[5] BISWAS K, HE J Q, BLUM I D, et al. High-performance bulk thermoelectrics with all-scale hierarchical architectures. Nature, 2012, 489: 414-418.

[6] ZHAO L D, LO S H, ZHANG Y S, et al. Ultralow thermal conductivity and high thermoelectric figure of merit in SnSe crystals. Nature, 2014, 508: 373-377.

[7] CHEN Z W, JIAN Z Z, LI W, et al. Lattice dislocations enhancing thermoelectric $\mathrm{PbTe}$ in addition to band convergence. Advanced Materials, 2017, 29(23): 1606768-1-8.

[8] KIM S I, LEE K H, MUN H A, et al. Dense dislocation arrays embedded in grain boundaries for high-performance bulk thermoelectrics. Science, 2015, 348(6230): 109-114.

[9] CHEN Z W, ZHANG X Y, PEI Y Z. Manipulation of phonon transport in thermoelectrics. Advanced Materials, 2018, 30(17): 1705617-1-12.

[10] HE Y, DAY T, ZHANG T S, et al. High thermoelectric performance in non-toxic earth-abundant copper sulfide. Advanced Materials, 2014, 26(23): 3974-3978.

[11] LIU H L, SHI X, XU F F, et al. Copper ion liquid-like thermoelectrics. Nature Materials, 2012, 11: 422-425.

[12] VINING C B, LASKOW W, HANSON J O, et al. Thermoelectric properties of pressure-sintered $\mathrm{Si}_{0.8} \mathrm{Ge}_{0.2}$ thermoelectric alloys. Journal of Applied Physics, 1991, 69(8): 4333-4340.

[13] PEI Y Z, LALONDE A, IWANAGA S, et al. High thermoelectric figure of merit in heavy hole dominated PbTe. Energy \& Environmental Science, 2011, 4(6): 2085-2089.

[14] ZEVALKINK A, TOBERER E S, ZEIER W G., et al. $\mathrm{Ca}_{3} \mathrm{AlSb}_{3}$ : an inexpensive, non-toxic thermoelectric material for waste heat recovery. Energy \& Environmental Science, 2011, 4(2): 510-518.

[15] MAY A F, TOBERER E S, SARAMAT A, et al. Characterization and analysis of thermoelectric transport in n-type $\mathrm{Ba}_{8} \mathrm{Ga}_{16-x} \mathrm{Ge}_{30+x}$. Physical Review B, 2009, 80(12): 125205-1-12.

[16] COX C A, TOBERER E S, LEVCHENKO A A, et al. Structure, heat capacity, and high-temperature thermal properties of $\mathrm{Yb}_{14} \mathrm{Mn}_{1-x} \mathrm{Al}_{x} \mathrm{Sb}_{11}$. Chemistry of Materials, 2009, 21(7): 1354-1360.

[17] SLACK G A. The thermal conductivity of nonmetallic crystals. Solid State Physics, 1979, 34: 1-71.

[18] TOBERER E S, ZEVALKINK A, SNYDER G J. Phonon engineering through crystal chemistry. Journal of Materials Chemistry, 2011, 21(40): 15843-15852.

[19] WANG Y, HU Y J, FIRDOSY S A, et al. First-principles calculations of lattice dynamics and thermodynamic properties for $\mathrm{Yb}_{14} \mathrm{MnSb}_{11}$. Journal of Applied Physics, 2018, 123(4): 045102-1-10.

[20] BROWN S R, KAUZLARICH S M, GASCOIN F, et al. $\mathrm{Yb}_{14} \mathrm{MnSb}_{11}$ : new high efficiency thermoelectric material for power generation. Chemistry of Materials, 2006, 18(7): 1873-1877.

[21] CHEN Z, LI D C, DENG S P, et al. Thermoelectric properties and thermal stability of Bi-doped PbTe single crystal. Physica B: Condensed Matter, 2018, 538: 154-159.

[22] YING P J, LI X, WANG Y C, et al. Hierarchical chemical bonds contributing to the intrinsically low thermal conductivity in $\alpha-\mathrm{MgAgSb}$ thermoelectric materials. Advanced Functional Materials, 2016, 27(1): 1604145-1-8.

[23] LI J Q, LI L F, SONG S H, et al. High thermoelectric performance of $\mathrm{GeTe}-\mathrm{Ag}_{8} \mathrm{GeTe}_{6}$ eutectic composites. Journal of Alloys and Compounds, 2013, 565: 144-147.

[24] FUJIKANE M, KUROSAKI K, MUTA H, et al. Thermoelectric properties of $\mathrm{Ag}_{8} \mathrm{GeTe}_{6}$. Journal of Alloys and Compounds, 2005, 396(1): 280-282.

[25] HOU Y H, CHANG L S. Optimization on the figure-of-merit of p-type $\mathrm{Ba}_{8} \mathrm{Ga}_{16} \mathrm{Ge}_{30}$ type-I clathrate grown via the Bridgman 
method by fine tuning $\mathrm{Ga} / \mathrm{Ge}$ ratio. Journal of Alloys and Compounds, 2018, 736: 108-114.

[26] YAN X L, IKEDA M, ZHANG L, et al. Suppression of vacancies boosts thermoelectric performance in type-I clathrates. Journal of Materials Chemistry A, 2018, 6(4): 1727-1735.

[27] BEEKMAN M, VANDERGRAAFF A. High-temperature thermal conductivity of thermoelectric clathrates. Journal of Applied Physics, 2017, 121(20): 205105.

[28] GONZALEZ-ROMERO R L, ANTONELLI A. Estimating carrier relaxation times in the $\mathrm{Ba}_{8} \mathrm{Ga}_{16} \mathrm{Ge}_{30}$ clathrate in the extrinsic regime. Physical Chemistry Chemical Physics, 2017, 19(4): 3010-3018.

[29] CHRISTENSEN M, ABRAHAMSEN A B, CHRISTENSEN N B, et al. Avoided crossing of rattler modes in thermoelectric materials. Nature Materials, 2008, 7: 811-815.

[30] CALLAWAY J. Model for lattice thermal conductivity at low temperatures. Physical Review, 1959, 113(4): 1046-1051.

[31] CHUNG J D, MCGAUGHEY A J H, KAVIANY M. Role of phonon dispersion in lattice thermal conductivity modeling. Journal of Heat Transfer, 2004, 126(3): 376-380.

[32] SLACK G A, GALGINAITIS S. Thermal conductivity and phonon scattering by magnetic impurities in CdTe. Physical Review, 1964, 133(1A): A253-A268.

[33] HEREMANS J P. Thermoelectric materials: the anharmonicity blacksmith. Nature Physics, 2015, 11: 990-991.

[34] QIU W J, XI L L, WEI P, et al. Part-crystalline part-liquid state and rattling-like thermal damping in materials with chemical-bond hierarchy. Proceedings of the National Academy of Sciences, 2014, 111(42): 15031-15035.

[35] TYAGI K, GAHTORI B, BATHULA S, et al. Thermoelectric properties of $\mathrm{Cu}_{3} \mathrm{SbSe}_{3}$ with intrinsically ultralow lattice thermal conductivity. Journal of Materials Chemistry A, 2014, 2(38): $15829-15835$.

[36] DELAIRE O, MA J, MARTY K, et al. Giant anharmonic phonon scattering in PbTe. Nature Materials, 2011, 10: 614-619.

[37] LEE S, ESFARJANI K, LUO T F, et al. Resonant bonding leads to low lattice thermal conductivity. Nature Communications, 2014, 5: 3525-1-8.

[38] MURPHY R M, MURRAY ÉD, FAHY S, et al. Ferroelectric phase transition and the lattice thermal conductivity of $\mathrm{Pb}_{1-x} \mathrm{Ge}_{x} \mathrm{Te}$ alloys. Physical Review B, 2017, 95(14): 144302-1-8.

[39] CHEN Y, HE B, ZHU T J, et al. Thermoelectric properties of non-stoichiometric $\mathrm{AgSbTe}_{2}$ based alloys with a small amount of GeTe addition. Journal of Physics D: Applied Physics, 2012, 45(11): 115302.

[40] ZHANG Y, KE X Z, CHEN C F, et al. Thermodynamic properties of $\mathrm{PbTe}, \mathrm{PbSe}$, and $\mathrm{PbS}$ : first-principles study. Physical Review B, 2009, 80(2): 024304-1-12.

[41] MILLER A J, SAUNDERS G A, YOGURTCU Y K. Pressure dependences of the elastic constants of PbTe, SnTe and $\mathrm{Ge}_{0.08} \mathrm{Sn}_{0.92} \mathrm{Te}$. Journal of Physics C: Solid State Physics, 1981, 14(11): $1569-1584$.

[42] ALEXANDER F Z, VOLKER L D, RALF P S, et al. Ab initio lattice dynamics and thermochemistry of layered bismuth telluride $\left(\mathrm{Bi}_{2} \mathrm{Te}_{3}\right)$. Journal of Physics: Condensed Matter, 2016, 28(11): 115401-1-7.

[43] RINCÓN C, VALERI-GIL M L, WASIM S M. Room-temperature thermal conductivity and grüneisen parameter of the I-III-VI chalcopyrite compounds. Physica Status Solidi (A), 1995, 147(2): 409-415.

[44] WANG H F, JIN H, CHU W G, et al. Thermodynamic properties of $\mathrm{Mg}_{2} \mathrm{Si}$ and $\mathrm{Mg}_{2} \mathrm{Ge}$ investigated by first principles method. Journal of Alloys and Compounds, 2010, 499(1): 68-74.

[45] BERNSTEIN N, FELDMAN J L, SINGH D J. Calculations of dynamical properties of skutterudites: thermal conductivity, thermal expansivity, and atomic mean-square displacement. Physical Re- view $B, 2010,81(13)$ : 134301-1-11.

[46] BHASKAR A, PAI Y H, WU W M, et al. Low thermal conductivity and enhanced thermoelectric performance of nanostructured Al-doped ZnTe. Ceramics International, 2016, 42(1, Part B): 1070-1076.

[47] KATRE A, TOGO A, TANAKA I, et al. First principles study of thermal conductivity cross-over in nanostructured zinc-chalcogenides. Journal of Applied Physics, 2015, 117(4): 045102-1-6.

[48] NUNES O A C. Piezoelectric surface acoustical phonon amplification in graphene on a GaAs substrate. Journal of Applied Physics, 2014, 115(23): 233715-1-7.

[49] REEBER R R. Thermal expansion of some group IV elements and ZnS. Physica Status Solidi (a), 1975, 32(1): 321-331.

[50] QIN L, TEO K L, SHEN Z X, et al. Raman scattering of Ge/Si dot superlattices under hydrostatic pressure. Physical Review B, 2001, 64(7): 075312-1-5.

[51] SILPAWILAWAN W, KUROSAKI K, OHISHI Y, et al. FeNbSb p-type half-Heusler compound: beneficial thermomechanical properties and high-temperature stability for thermoelectrics. Journal of Materials Chemistry C, 2017, 5(27): 6677-6681.

[52] BOSONI E, SOSSO G C, BERNASCONI M. Grüneisen parameters and thermal conductivity in the phase change compound GeTe. Journal of Computational Electronics, 2017, 16 (4): 997-1002.

[53] LI W, LIN S, GE B, et al. Low sound velocity contributing to the high thermoelectric performance of $\mathrm{Ag}_{8} \mathrm{SnSe}_{6}$. Advanced Science, 2016, 3 (11): 1600196-1-7.

[54] CALLAWAY J, VON B, HANS C. Effect of point imperfections on lattice thermal conductivity. Physical Review, 1960, 120(4): $1149-1154$.

[55] HAO F, QIU P F, TANG Y S, et al. High efficiency $\mathrm{Bi}_{2} \mathrm{Te}_{3}$-based materials and devices for thermoelectric power generation between 100 and $300{ }^{\circ} \mathrm{C}$. Energy \& Environmental Science, 2016, 9(10): 3120-3127.

[56] HU L P, ZHU T J, LIU X H, et al. Point defect engineering of high-performance bismuth-telluride-based thermoelectric materials. Advanced Functional Materials, 2014, 24(33): 5211-5218.

[57] PEI Y Z, SHI X Y, LALONDA A, et al. Convergence of electronic bands for high performance bulk thermoelectrics. Nature, 2011, 473(7345): 66-69.

[58] QIN Y T, QIU P F, SHI X, et al. Thermoelectric properties for Cu$\operatorname{InTe}_{2-x} \mathrm{~S}_{x}(x=0,0.05,0.1,0.15)$ solid solution. Journal of Inorganic Materials, 2017, 32(11): 1171-1176.

[59] JIANG G Y, HE J, ZHU T J, et al. High performance $\mathrm{Mg}_{2}(\mathrm{Si}, \mathrm{Sn})$ solid solutions: a point defect chemistry approach to enhancing thermoelectric properties. Advanced Functional Materials, 2014, 24(24): 3776-3781.

[60] LIU X H, ZHU T J, WANG H, et al. Low electron scattering potentials in high performance $\mathrm{Mg}_{2} \mathrm{Si}_{0.45} \mathrm{Sn}_{0.55}$ based thermoelectric solid solutions with band convergence. Advanced Energy Materials, 2013, 3(9): 1238-1244.

[61] TRIPATHI M N, BHANDARI C M. High-temperature thermoelectric performance of Si-Ge alloys. Journal of Physics: Condensed Matter, 2003, 15(31): 5359-5370.

[62] FU C G, ZHU T J, PEI Y Z, et al. High band degeneracy contributes to high thermoelectric performance in p-type half-Heusler compounds. Advanced Energy Materials, 2014, 4(18): 1400600 $1-6$.

[63] YU J J, XIA K Y, ZHAO X B, et al. High performance p-type half-Heusler thermoelectric materials. Journal of Physics D: Applied Physics, 2018, 51(11): 113001.

[64] SHEN J J, FU C G, LIU Y T, et al. Enhancing thermoelectric performance of $\mathrm{FeNbSb}$ half-Heusler compound by Hf-Ti dual-doping. Energy Storage Materials, 2018, 10: 69-74.

[65] ZHU T J, LIU Y T, FU C G, et al. Compromise and synergy in high-efficiency thermoelectric materials. Advanced Materials, 
2017, 29(14): 1605884-1-26.

[66] FU C G, WU H J, LIU Y T, et al. Enhancing the figure of merit of heavy-band thermoelectric materials through hierarchical phonon scattering. Advanced Science, 2016, 3(8): 1600035-1-6.

[67] ZHU T J, FU C G, XIE H H, et al. High efficiency half-Heusler thermoelectric materials for energy harvesting. Advanced Energy Materials, 2015, 5(19): 1500588-1-7.

[68] FU C G, BAI S Q, LIU Y T, et al. Realizing high figure of merit in heavy-band p-type half-Heusler thermoelectric materials. Nat. Commun., 2015, 6: 8144 .

[69] XIE H H, WANG H, PEI Y Z, et al. Beneficial contribution of alloy disorder to electron and phonon transport in half-heusler thermoelectric materials. Advanced Functional Materials, 2013, 23(41): 5123-5130.

[70] YU J J, FU C G, LIU Y T, et al. Unique role of refractory ta alloying in enhancing the figure of merit of $\mathrm{NbFeSb}$ thermoelectric materials. Advanced Energy Materials, 2018, 8(1): 1701313-1-8.

[71] XIA K Y, LIU Y T, ANAND S, et al. Enhanced thermoelectric performance in 18-electron $\mathrm{Nb}_{0.8} \mathrm{CoSb}$ half-heusler compound with intrinsic Nb vacancies. Advanced Functional Materials, 2018, 28(9): 1705845-1-7.

[72] LI W, LIN S Q, ZHANG X Y, et al. Thermoelectric properties of $\mathrm{Cu}_{2} \mathrm{SnSe}_{4}$ with intrinsic vacancy. Chemistry of Materials, 2016, 28(17): 6227-6232.

[73] KLEMENS P G. The scattering of low-frequency lattice waves by static imperfections. Proceedings of the Physical Society. Section A, 1955, 68(12): 1113-1128.

[74] ZHANG S N, HE J, JI X H, et al. Effects of ball-milling atmosphere on the thermoelectric properties of TAGS-85 compounds. Journal of Electronic Materials, 2009, 38(7): 1142-1147.

[75] LI Y, MEI D Q, WANG H, et al. Reduced lattice thermal conductivity in nanograined $\mathrm{Na}$-doped $\mathrm{PbTe}$ alloys by ball milling and semisolid powder processing. Materials Letters, 2015, 140: 103-106.

[76] HONG M, CHEN Z G, ZOU J. Fundamental and progress of $\mathrm{Bi}_{2} \mathrm{Te}_{3}$-based thermoelectric materials. Chinese Physics B, 2018, 27(4): 048403-1-46.

[77] XIE J, OHISHI Y, ICHIKAWA S, et al. Naturally decorated dislocations capable of enhancing multiple-phonon scattering in Si-based thermoelectric composites. Journal of Applied Physics, 2018, 123(11): 115114-1-8.

[78] YU Y, HE D S, ZHANG S Y, et al. Simultaneous optimization of electrical and thermal transport properties of $\mathrm{Bi}_{0.5} \mathrm{Sb}_{1.5} \mathrm{Te}_{3}$ thermoelectric alloy by twin boundary engineering. Nano Energy, 2017, 37: 203-213.

[79] XIE W J, HE J, KANG H J, et al. Identifying the specific nanostructures responsible for the high thermoelectric performance of $(\mathrm{Bi}, \mathrm{Sb})_{2} \mathrm{Te}_{3}$ nanocomposites. Nano Letters, 2010, 10(9): 3283-3289.

[80] YANG X Y, WU J H, REN D D, et al. Microstructure and thermoelectric properties of p-type $\mathrm{Si}_{80} \mathrm{Ge}_{20} \mathrm{~B}_{0.6}-\mathrm{SiC}$ nanocomposite. Journal of Inorganic Materials, 2016, 31(9): 997-1003.

[81] YU C, XIE H H, FU C G, et al. High performance half-Heusler thermoelectric materials with refined grains and nanoscale precipitates. Journal of Materials Research, 2012, 27(19): 2457-2465.

[82] HU L P, WU H J, ZHU T J, et al. Tuning multiscale microstructures to enhance thermoelectric performance of n-type bismuth-telluride-based solid solutions. Advanced Energy Materials, 2015, 5(17): 1500411-1-13.

[83] HE J Q, GIRARD S N, KANATZIDIS M G, et al. Microstructure-lattice thermal conductivity correlation in nanostructured $\mathrm{PbTe}_{0.7} \mathrm{~S}_{0.3}$ thermoelectric materials. Advanced Functional Materials, 2010, 20(5): 764-772.

[84] XIN J Z, WU H J, LIU X H, et al. Mg vacancy and dislocation strains as strong phonon scatterers in $\mathrm{Mg}_{2} \mathrm{Si}_{1-x} \mathrm{Sb}_{x}$ thermoelectric materials. Nano Energy, 2017, 34: 428-436.

[85] SHI X, BAI S Q, XI L L, et al. Realization of high thermoelectric performance in n-type partially filled skutterudites. Journal of Materials Research, 2011, 26(15): 1745-1754.

[86] KEPPENS V, MANDRUS D, SALES B C, et al. Localized vibrational modes in metallic solids. Nature, 1998, 395: 876-878.

[87] DUAN B, YANG J, SALVADOR J R, et al. Electronegative guests in $\mathrm{CoSb}_{3}$. Energy \& Environmental Science, 2016, 9(6): 2090-2098.

[88] SAMANTA M, PAL K, PAL P, et al. Localized vibrations of bi bilayer leading to ultralow lattice thermal conductivity and high thermoelectric performance in weak topological insulator n-type BiSe. Journal of the American Chemical Society, 2018, 140(17): 5866-5872.

[89] UHER C, YANG J, HU S, et al. Transport properties of pure and doped MNiSn (M=Zr, Hf). Physical Review B, 1999, 59(13): 8615-8621.

[90] LI J F, LIU W S, ZHAO L D, et al. High-performance nanostructured thermoelectric materials. NPG Asia Materials, 2010, 2(4): 152-158.

[91] FANG T, ZHAO X B, ZHU T J. Band Structures and transport properties of high-performance half-heusler thermoelectric materials by first principles. Materials, 2018, 11(5): 847 .

[92] TANG Y L, LI X S, MARTIN L H J, et al. Impact of Ni content on the thermoelectric properties of half-Heusler TiNiSn. Energy \& Environmental Science, 2018, 11(2): 311-320.

[93] ZHU T J, YU G T, XU J, et al. The role of electron-phonon interaction in heavily doped fine-grained bulk silicons as thermoelectric materials. Advanced Electronic Materials, 2016, 2(8): 1600171.

[94] ABELES B. Lattice thermal conductivity of disordered semiconductor alloys at high temperatures. Physical Review, 1963, 131(5): 1906-1911.

[95] CLARKE D R. Materials selection guidelines for low thermal conductivity thermal barrier coatings. Surface and Coatings Technology, 2003, 163: 67-74.

[96] CAHILL D G, POHL R O. Heat flow and lattice vibrations in glasses. Solid State Communications, 1989, 70(10): 927-930.

[97] CAHILL D G, WATSON S K, POHL R O. Lower limit to the thermal conductivity of disordered crystals. Physical Review B, 1992, 46(10): 6131-6140.

[98] ALLEN P B, DU X Q, MIHALY L, et al. Thermal conductiity of insulating $\mathrm{Bi}_{2} \mathrm{Sr}_{2} \mathrm{YCu}_{2} \mathrm{O}_{8}$ and superconducting $\mathrm{Bi}_{2} \mathrm{Sr}_{2} \mathrm{CaCu}_{2} \mathrm{O}_{8}$ : failure of the phonon-gas picture. Physical Rview B, 1994, 49(13): 9073-9079.

[99] FELDMAN J L, ALLEN P B, BICKHAM S R. Numerical study of low-frequency vibrations in amorphous silicon. Physical Review B, 1999, 59(5): 3551-3559.

[100] AGNE M T, HANUS R, SNYDER G J. Minimum thermal conductivity in the context of diffuson-mediated thermal transport. Energy \& Environmental Science, 2018, 11(3): 609-616.

[101] POHL R O. Lattice vibrations of glasses. Journal of Non-Crystalline Solids, 2006, 352(32): 3363-3367.

[102] FU C G, ZHU T J, LIU Y T, et al. Band engineering of high performance p-type FeNbSb based half-Heusler thermoelectric materials for figure of merit $z T>1$. Energy \& Environmental Science, 2015, 8(1): 216-220.

[103] WEI P, YANG J, GUO L, et al. Minimum thermal conductivity in weak topological insulators with bismuth-based stack structure. Advanced Functional Materials, 2016, 26(29): 5360-5367.

[104] RASCHE B, ISAEVA A, RUCK M, et al. Stacked topological insulator built from bismuth-based graphene sheet analogues. Nature Materials, 2013, 12(5): 422-425.

[105] PAULY C, RASCHE B, KOEPERNIK K, et al. Subnanometre-wide electron channels protected by topology. Nature Physics, 2015, 11(4): 338-343. 